THEOREM 5. A space satisfies the first enumerability axiom if each point $p$ has a well ordered decreasing family of neighborhoods equivalent to $\left[U_{p}\right]$, the sets $L(E)$ are closed, and either every set is separable or the space is regular and every set is condensed in itself.

From this result we can get variations of Theorems 3 and 4 .

6. An Example. Frink proposed to me the problem (which he and Hildebrandt had considered) of constructing a bicompact separable, completely normal, Hausdorff space, satisfying the first enumerability axiom but not the second. The circumference of a circle is such a space if we define as follows the sets which for every point $p$ have $p$ as a limit point. Any set $E$ has $p$ as a limit point if and only if there are points of $E-p$ either (1), arbitrarily near $p$ in the direction clockwise from $p$, or (2), arbitrarily near $q$ (the point opposite $p$ ) in the direction counterclockwise from $q$. If we omit (2), we have a locally non-compact space having the other properties mentioned. $\dagger$

UNIVERSITY OF IOWA

\title{
ADDENDUM ON FACTORABILITY OF NUMERICAL FUNCTIONS
}

BY E. T. BELL

It has been pointed out to me that the statement of the Lemma on page 252 of the April, 1931, issue of this Bulletin might be misunderstood. To clear up any possible misunderstanding, it is sufficient to point out that the notation $f, g, h$ is as in the italicised statement on page 251 , as is evident on referring to paragraph 3 , third sentence. At the top of page 253, for "all or none of $f, g, \cdots, h$ are factorable," read "the only excluded possibility is that in which precisely one of $f, g, \cdots, h$ is not factorable." This is evident from paragraph 3 .

California Institute of Technology

$\dagger$ See Fréchet, Transactions of this Society, loc. cit., p. 60; and D. McCoy, loc. cit., p. 114 (S 241). 